\title{
Forbush decrease precursors observed in GRAPES-3
}

\author{
K. P. Arunbabu ${ }^{* a, b}$, H. M. Antia ${ }^{a, b}$, S. R. Dugad ${ }^{a, b}$, S. K. Gupta ${ }^{a, b}$, Y. Hayashi ${ }^{a, d}$, S. \\ Kawakami $^{a, d}$, P. K. Mohanty ${ }^{a, b}$, A. Oshima ${ }^{a, e}$, P. Subramanian ${ }^{a, c}$ \\ ${ }^{a}$ The GRAPES-3 Experiment, Cosmic Ray Laboratory, Raj Bhavan, Ooty 643 001, India \\ ${ }^{b}$ Tata Institute of Fundamental Research, Homi Bhabha Road, Mumbai 400 005, India \\ ${ }^{c}$ Indian Institute of Science Education and Research, Dr. Homi Bhabha Road, Pune 411 021, \\ India \\ ${ }^{d}$ Graduate School of Science, Osaka City University, Osaka 558-8585, Japan \\ ${ }^{e}$ College of Engineering, Chubu University, Kasugai, Aichi 487-8501, Japan \\ E-mail: arun.babu@tifr.res.in
}

\begin{abstract}
Earth-directed Coronal mass ejections (CMEs) emanating from the Sun and the shock associated with it are the primary drivers of space weather disturbances. Forbush decrease precursors are advance warning of these upcoming magnetic field disturbances. GRAPES-3 tracking muon telescope which is a part of GRAPES-3 experiment located in Ooty, India, provides high statistics measurement of the muon flux with good temporal resolution. In this study we are using data from GRAPES-3 muon telescope and making use of its multidirectional observations to study the Forbush decrease precursors in greater detail. We have identified few Forbush decrease precursor signatures in muon flux well before the arrival of the actual shock. We can use these Forbush decrease precursors to study the characteristics of magnetic field compression associated with the upcoming CME shock-sheath system.
\end{abstract}

The 34th International Cosmic Ray Conference,

30 July- 6 August, 2015

The Hague, The Netherlands

\footnotetext{
* Speaker.
} 


\section{Introduction}

It is known that Earth directed coronal mass ejections (CMEs) and its shock forms a reductions in cosmic ray intensity observed in Earth during its passage, which is known as Forbush decreases (FD) $[1,2]$. These FDs are caused due to the magnetic field compressions associated with the CME-shock system, which is engulfing Earth. Some anisotropies in cosmic ray intensity is observed before the arrival of shock, because, the cosmic rays travel much faster than the CME-shock system, and it carry the information about the depleted region to Earth far upstream of the approaching CME/shock system. These anisotropy in cosmic ray intensity before the arrival of shock are known as precursors. Some times these precursory anisotropy is observed as the "loss cone" (LC) anisotropy, which is characterized by an intensity deficit in cosmic ray intensity confined to a narrow pitch angle region around the sunward interplanetary magnetic field (IMF) direction $[3,4]$. These Forbush decrease precursors are advanced warning of upcoming shock/CME which can be observed much before the actual arrival of shock. FD precursors observed with a long lead time are of particular importance for the possibility of space weather predictions using cosmic ray measurements.

In this work we are explaining example of FD precursor events observed in GRAPES-3 muon telescope. This works shows the statistical and temporal advantage of this instrument which can observe precursors in much more details in 169 directions along the $2.2 \mathrm{sr}$ field of view.

\section{GRAPES-3 muon telescope}

The GRAPES-3 experiment is located at Ooty $\left(11.4^{\circ} \mathrm{N}\right.$ latitude, $76.7^{\circ} \mathrm{E}$ longitude, and $2200 \mathrm{~m}$ altitude) in India. This experiment contains two major components, first an air shower array of 400 scintillation detectors (each of $1 \mathrm{~m}^{2}$ ) arranged in a hexagonal geometry, with a distance of $8 \mathrm{~m}$ between adjacent detectors $[5,6,7,8]$. The second component of is a large area tracking muon telescope. This muon telescope is a unique instrument used to search for high energy protons emitted during the active phase of a solar flare or a CME, which provides a high statistics, directional measurement of the muon flux. This telescope covers an area of $560 \mathrm{~m}^{2}$, consisting of a total of 16 modules, each of them is $35 \mathrm{~m}^{2}$ in area. The energy threshold of the telescope is $\sec (\theta) \mathrm{GeV}$, for the muons arriving along a direction with zenith angle $\theta$. The observed muon rate of $\sim 3000 \mathrm{~s}^{-1}$ per module, yields a total muon rate $\sim 3 \times 10^{6} \mathrm{~min}^{-1}$ for the entire telescope $[9,10]$. This large rate permits even a small change of $\lesssim 0.1 \%$ in the muon flux to be measured accurately over a time scale of $\sim 5 \mathrm{~min}$, after appropriate corrections are applied for the time dependent variation in the atmospheric pressure [11]. The design of the telescope help us to look in 169 solid angle directions [10, 12], also these 169 directions were regrouped in a suitable manner to form nine different directions, named NW (northwest), N (north), NE (northeast), W (west), V (vertical), E (east), SW (southwest), S (south), and SE (southeast) with uniform solid angle in field of view [10]. The cutoff rigidity due to the geomagnetic field at Ooty along the vertical direction is $17 \mathrm{GV}$ and it varies from 14 to $42 \mathrm{GV}$ across the 2.2 sr. field of view of the muon telescope. Details of the muon telescope are given in $[9,10,2]$. 


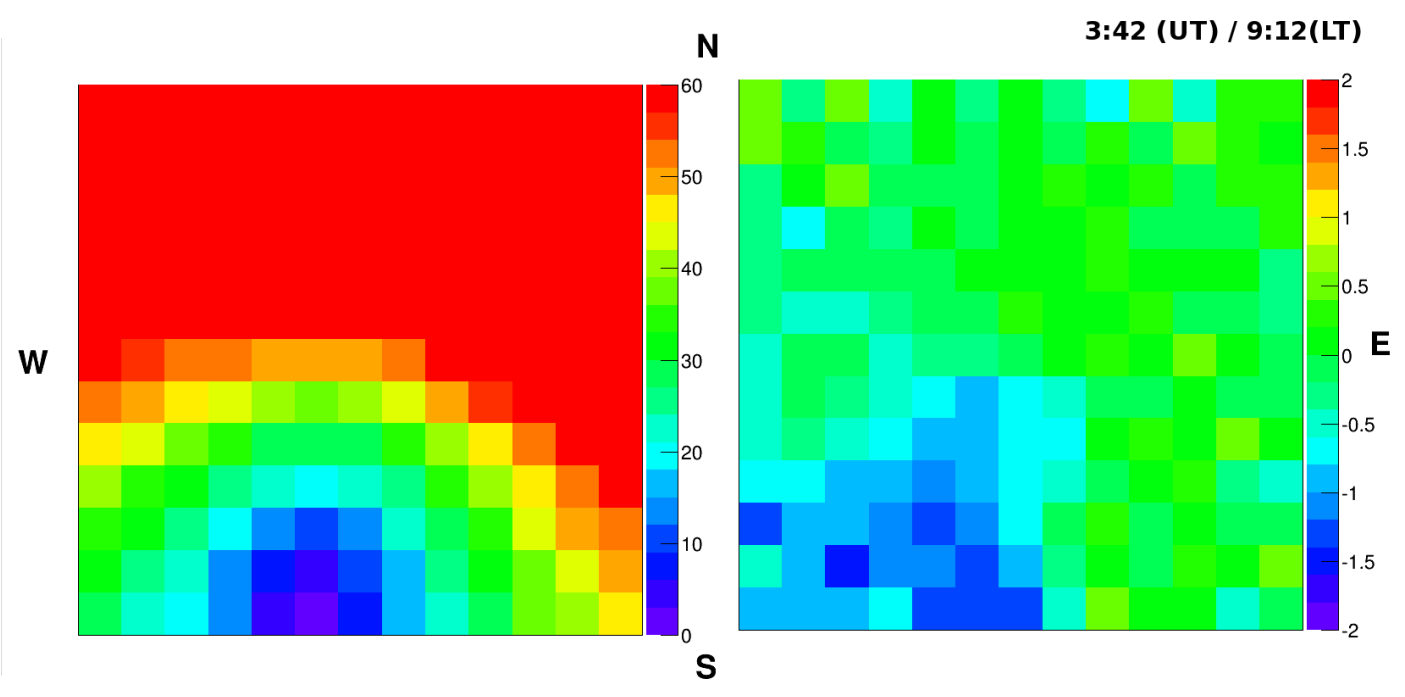

Figure 1: Forbush decrease precursor observed in GRAPES-3 at 9:12 local time or 3:42 UT. first figure shows the pitch angle of the viewing directions of GRAPES-3 muon telescope, second panel shows the percentage deviation of observed muon flux

\section{Data analysis}

In this work we analyzed the 4-minute average muon flux data from the GRAPES-3 muon telescope. We had applied the efficiency correction to the GRAPES-3 data for the 16 modules. We then applied the pressure correction to this data. The Data after going through efficiency correction and pressure correction will represent the actual cosmic ray intensity reaching our upper atmosphere. This data will have the signatures of interplanetary magnetic field modulation on cosmic rays and diurnal variations. Since we are interested in Forbush decrease events and its precursors we used a band-rejection-filter to remove the periodic diurnal variations and its second harmonics. The filter we used removes all the frequencies in the range $1 /$ day $\leq f \leq 2 /$ day. After applying the filter to the muon flux data, the diurnal variations and its second harmonics are removed and contain mainly signatures of solar activities.

We calculated the percentage deviation of muon flux from average value in the quite time period. Forbush decrease precursors shows significant change in the intensity well before the arrival of shock. We identified this changes in intensity in 169 directions and as well as in nine directions. to confirm that the observed variations are connected with the up coming shock/CME we checked the pitch angle of our directions with the directions of interplanetary magnetic field. We took the GSE coordinate IMF data from ACE/WIND space crafts corresponding to the time of observed precursor decreases and calculated the pitch angle. Since precursors are caused due to the presence of earth directed CME-shock system magnetically connected Sun-Earth line, we can see the precursors in lowest pitch angles only $[3,4]$. One example of the FD precursor observed in 169 directions and the pitch angle of corresponding viewing directions at 9:12 local time or 3:42 UT is shown in figure 1. For a simpler understand of our observational directions and pitch angle comparison, we are presenting the precursor events in Ooty local time (LT = UT+5:30 hrs), which is 5 hrs 30 minute ahead of UT. 


\section{FD precursors observed in GRAPES-3}

We identified Forbush decrease precursors well before arrival of shock. With the high statistics and temporal resolution of GRAPES-3 data we identified the FD precursor events in details.

\subsection{FD precursor on 14 December 2006}

This event was observed on 14 December 2006, followed by a FD. This event was associated with a CME which was first observed in LASCO field of view on 13 December 2006 at 02:54 UT, which was having a speed of $\sim 1774 \mathrm{~km} \mathrm{~s}^{-1}$. The shock associated with this CME reached Earth and identified by ACE/WIND spacecrafts on 14 December 2006 at 14:14 UT, which corresponds to 19:44 LT, the solar wind speed was $\sim 955 \mathrm{~km} \mathrm{~s}^{-1}$. Figure 2 shows the precursors event observed in South-East direction. First panel shows the percentage deviation of muon flux in SE direction and second panel shows the scalar magnetic field data observed in ACE/WIND spacecrafts available from OMNI database. In this direction the precursor event was observed more than $10 \mathrm{hrs}$ before the arrival of shock, which is represented by blue vertical line in figure 2 and 3.

The precursors started appearing initially in North-East directions and gradualy moved towards South-West directions. This event observed in nine different direction are shown in figure 3, and the evolution of this precursor event in 169 direction of GRAPES-3 field of view is represented in figure 4.

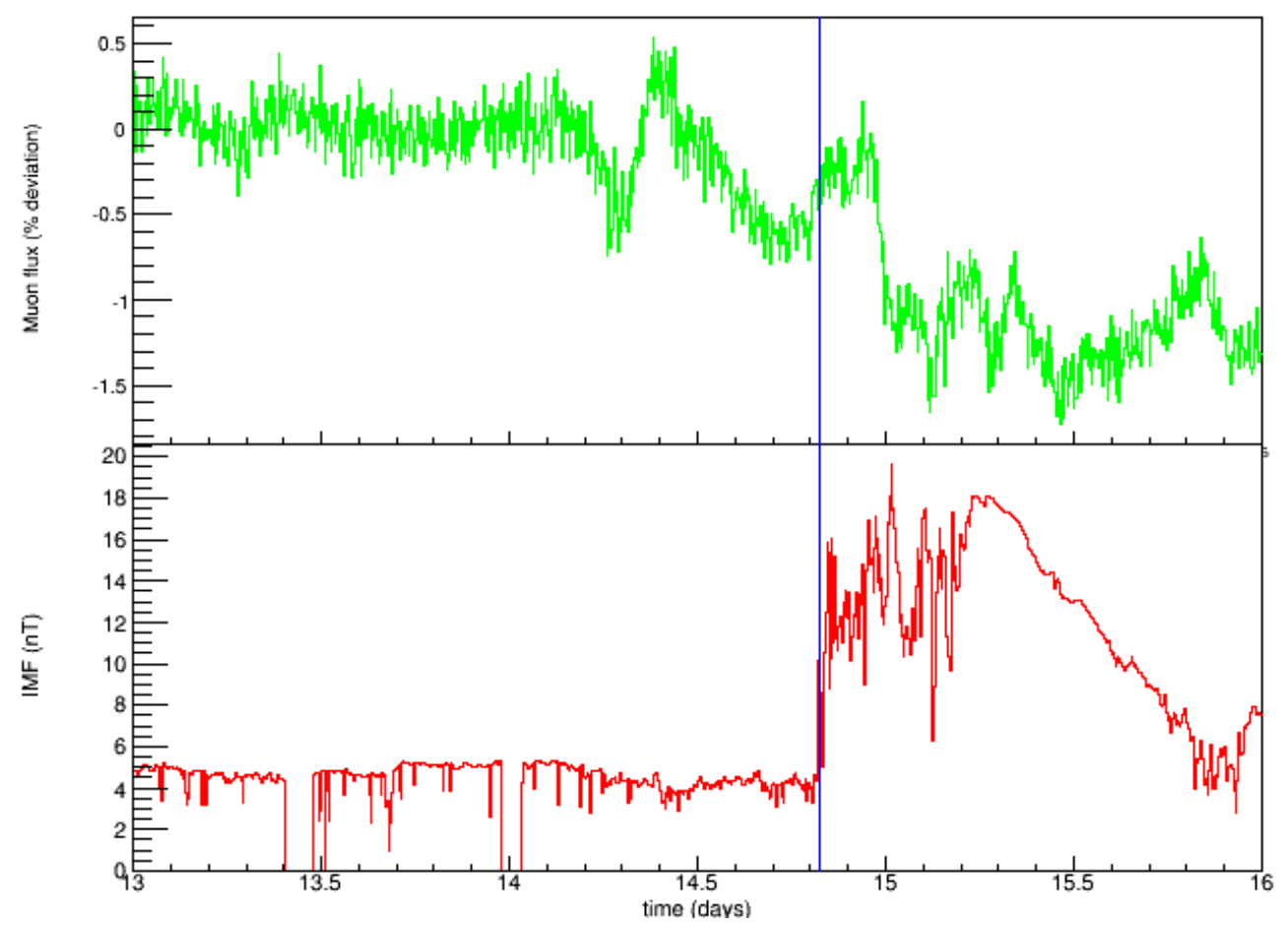

Figure 2: Top panel shows the percentage deviation of muonflux observed in SE direction. Bottom panel shows the scalar interplanetary magnetic field. Vertical line corresponds to the shock arrival time. The time is given in Ooty local time (LT=UT+5:30 hrs). 


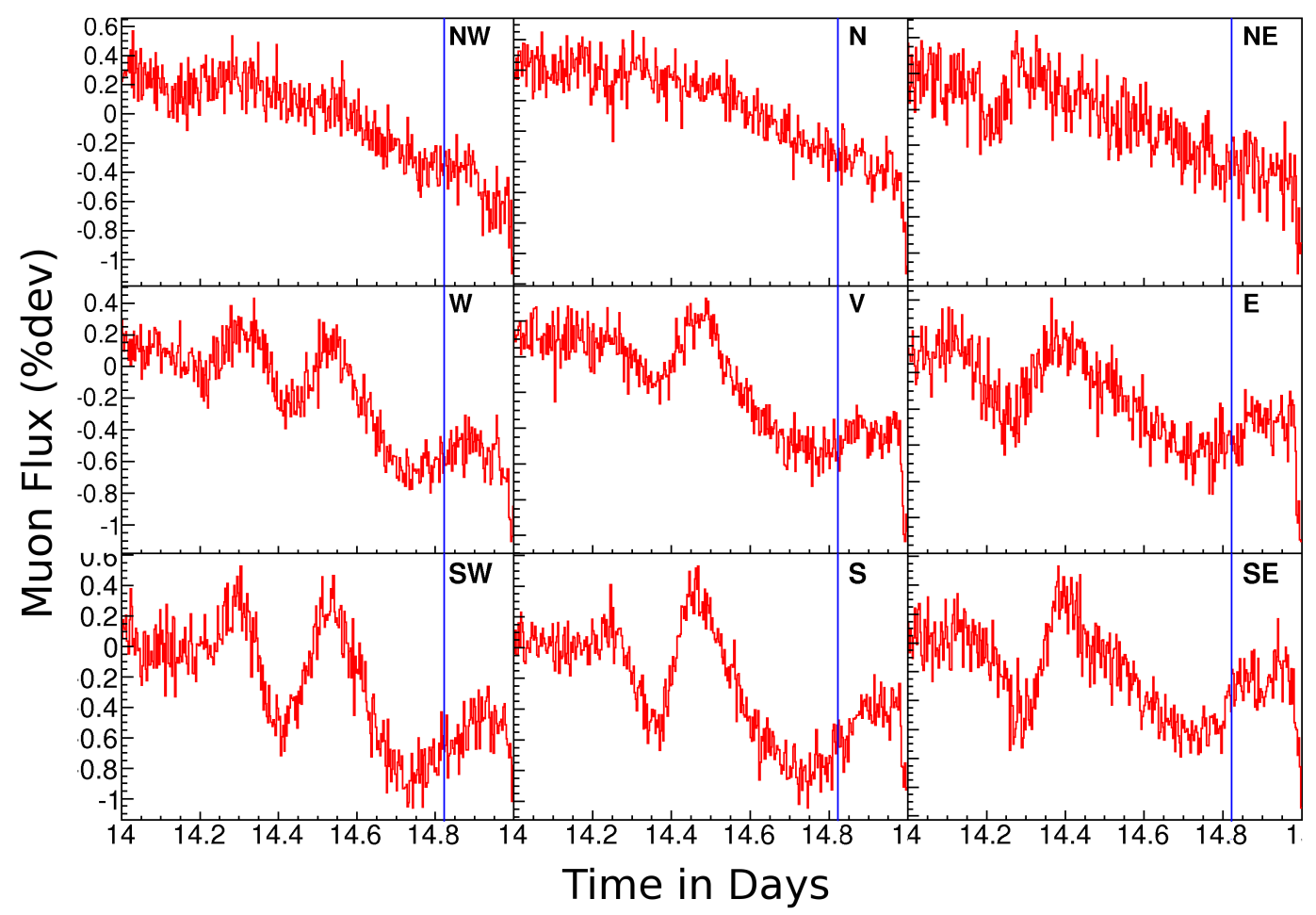

Figure 3: FD precursors on 14 December 2006 observed in nine different directions using GRAPES-3 muon telescope. Each panel shows the percentage deviation of muon flux and the vertical line shows the shock arrival time. The time is given in Ooty local time ( $\mathrm{LT}=\mathrm{UT}+5: 30 \mathrm{hrs})$.

\subsection{FD precursor on 29 October 2003}

This event was observed on 29 October 2003 followed by the largest FD observed in $23^{\text {rd }}$ Solar cycle. This event was associated with a CME that observed first on 28 October 2003 at 11:30 UT with a speed $\sim 2459 \mathrm{~km} \mathrm{~s}^{-1}$, which caused the famous Halloween events. The shock associated with this event reached Earth on 29 October at 06:11 UT, which correspond to 11:41 Ooty local time. The solar wind speed during the arrival of shock/CME system was $\sim 1900 \mathrm{~km} \mathrm{~s}^{-1}$. We started seeing the precursors $\sim 7$ hours before the arrival of shock. This event observed in nine different direction are shown in figure 5, and the evolution of this precursor event in 169 direction of GRAPES-3 field of view is represented in figure 6.

\section{Conclusions}

GRAPES-3 muon telescope gives the muon flux data with high statistics and temporal resolution. We designed a band rejections filter which removes the frequencies in the range $1 /$ day $\leq$ $f \leq 2 /$ day. this helped us to removed the components of diurnal anisotropy and its first harmonics. The resultant data after applying the filter contains the Forbush decrease and precursor event clearly. We identified Forbush decrease precursors well before the arrival of actual shock in more detail. The evolution of precursor events in the 169 directions FOV can give us informations about the upcoming CME, which can help us to understand the precursors. 

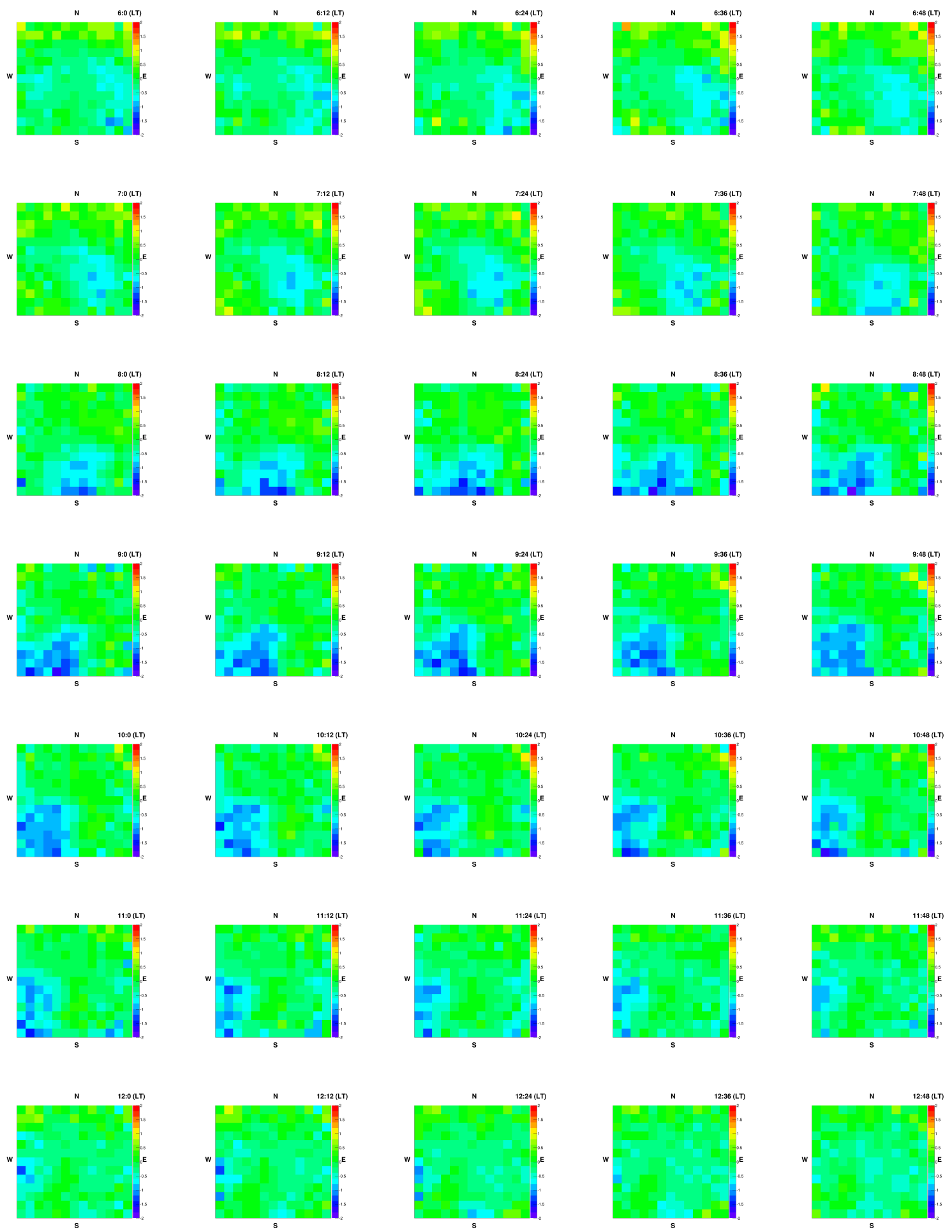

Figure 4: Forbush decrease precursors observed in 169 directions on 14 December 2006. The time is given in Ooty local time (LT=UT+5:30 hrs). 


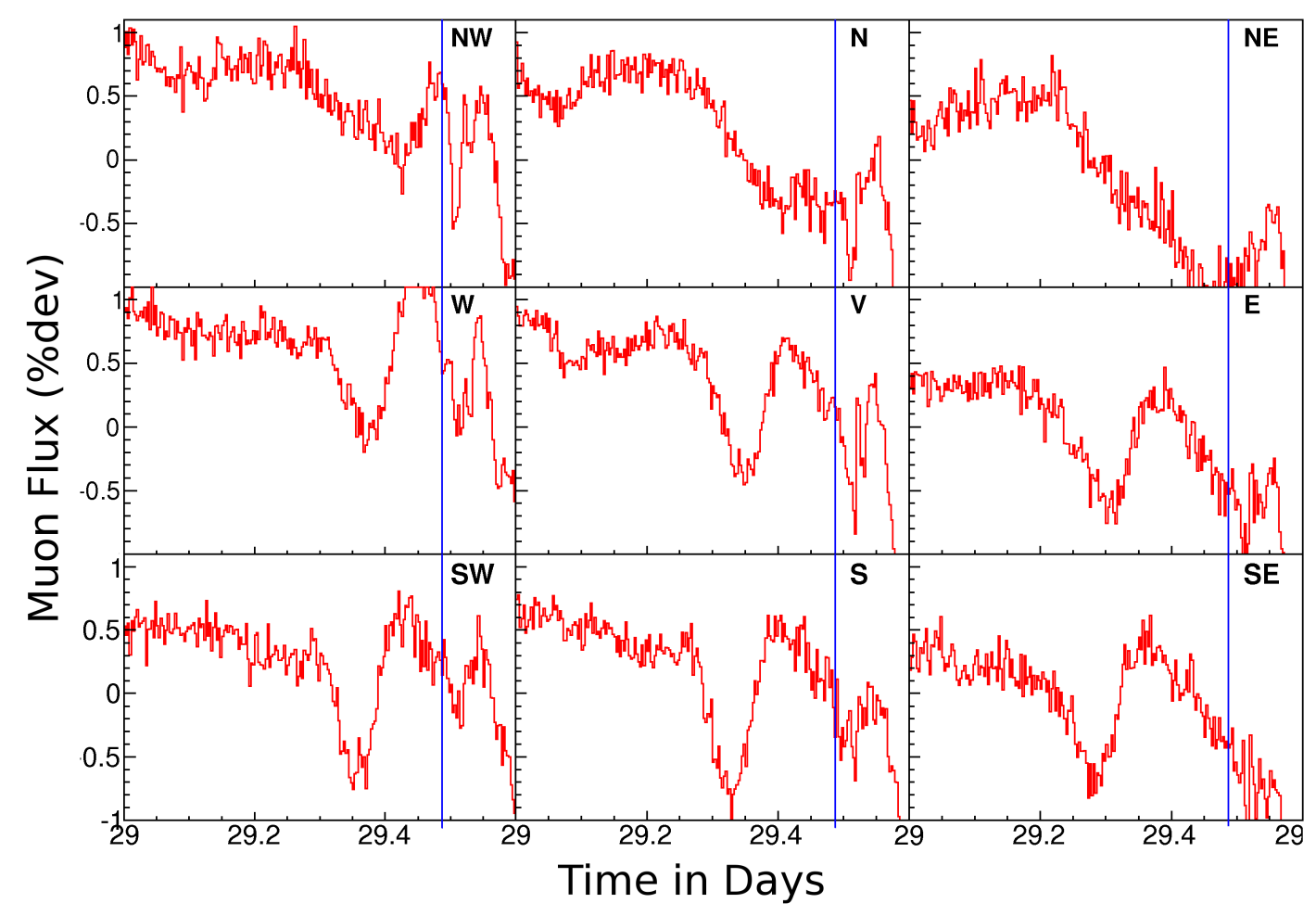

Figure 5: FD precursors on 29 October 2003 observed in nine different directions using GRAPES-3 muon telescope. Each panel shows the percentage deviation of muon flux and the vertical line shows the shock arrival time. The time is given in Ooty local time (LT=UT+5:30 hrs).

\section{References}

[1] Arunbabu, K. P., et. al., 2013, A\&A, 555A, 139A

[2] Subramanian, P., et.al, 2009, A\&A, 494, 1107

[3] Munakata, K. et. al, 2000, JGR, 105, 27457

[4] Munakata, K. et. al, 2005, GeoRL, 32, L03S04M

[5] Gupta, S. K., et. al., 2005, Nucl. Instrum. Methods A, 540, 311

[6] Gupta, S. K., et. al., 2009, Nucl. Phys. B Proc. Suppl., 196, 153

[7] Mohanty, P. K., et al., 2009, Astropart. Phys. 31, 24

[8] Mohanty, P. K., Dugad, S. R., \& Gupta, S. K., 2012, Rev. Sci. Instrum. 83, 043301

[9] Hayashi, Y., et al., 2005, Nucl. Instrum. Methods A, 545, 643

[10] Nonaka, T., et al., 2006, Phys. Rev. D., 74, 052003

[11] Mohanty, P. K., et al., 2013, Pramana J. Phys., 81, 343

[12] Kojima, H., et. al., 2015, Astroparticle Phys, 62, 21 

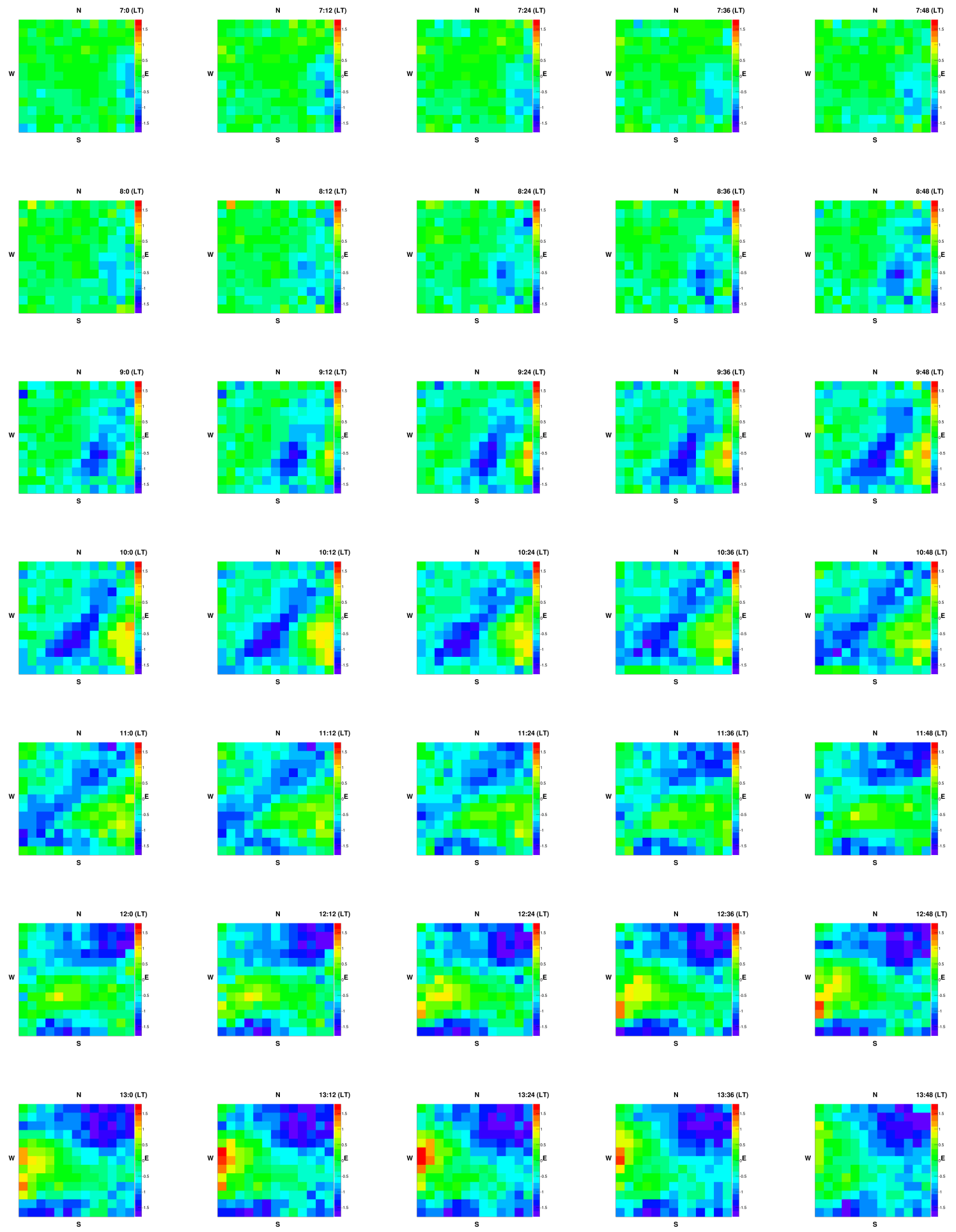

Figure 6: Forbush decrease precursors observed in 169 directions on 29 October 2003. The time is given in Ooty local time (LT=UT+5:30 hrs). 